\title{
Healthy debate on early Mars
}

To the Editor - In a recent Editorial ${ }^{1}$, it was suggested that early Mars research is 'at war' and that entrenched positions on either side are impeding progress. We disagree with this depiction of the Mars community. Instead, the Mars climate evolution debate is dynamic and intensely data-driven, and we are all striving for more. The past decade has seen remarkable progress in our understanding of Mars' early environment as a result of new rover data, new orbital observations and advances in climate modelling.

No one in the Mars community contests the idea that abundant liquid water flowed on Mars 3 to 4 billion years ago and perhaps more recently. However, given Mars' distance from the young Sun, which was probably also fainter than today, more than $60 \mathrm{~K}$ of greenhouse warming - around double the greenhouse effect on present-day Earth and nine times that on present-day Mars - is required to melt surface ice and create this water ${ }^{2,3}$. This makes permanently warm conditions extremely hard to achieve in realistic climate models. Given this, we need to consider both episodic and continuous warming mechanisms and pose the question how different early Mars must have been from the present day to explain the geological observations. This creates a rich, cross-disciplinary discussion where predictions from specific climate scenarios can be directly tested against the geologic record.

Fortunately, because over $50 \%$ of the surface of Mars dates from this crucial early period, its geologic record preserves the details of its climate transitions in remarkable detail. Indeed, Mars is a unique solar system archive of the early history of terrestrial planets that includes responses to crust formation, meteoroid bombardment and an evolving young sun. We have already learnt much from this archive, but far more remains to be uncovered in future.

The current robust debate over the nature of Mars' early climate is healthy, but the positive path forward to its resolution requires more data and better models. Three research areas are particularly critical: geomorphology, geochemistry and climate modelling. From geomorphic studies, better consensus needs to be acquired on the time required to carve valley networks ${ }^{4}$, form open- and closed-basin lakes and produce thick sedimentary deposits ${ }^{5}$. We also need to establish consensus on the requirements for rain or snowmelt as the source of liquid water, and the presence or absence of a Noachian ocean. The controls on and evidence for glaciation under different background climate scenarios ${ }^{6}$ need to be more fully explored. Better geochemical data on mineralogical alteration rates and on how observed products relate to a wider range of environmental conditions are also required.

\section{Mars is a unique solar system archive of the early history of terrestrial planets.}

From climate modelling, simulations with more realistic representation of cloud and convection physics are necessary, as are simulations that couple sub-surface hydrology to atmospheric processes. Further constraints from quantitative spectroscopy and radiative transfer modelling are needed to provide insight into the warming potential of various greenhouse gas combinations. Finally, more sophisticated models of atmospheric loss processes, particularly for species other than $\mathrm{CO}_{2}$, are also necessary.

Observationally, the most significant new constraints on the early Martian climate are likely to come from further in situ rover analysis and, eventually, analysis of samples returned to Earth. Specific goals of future Mars measurements should be the characterization of climatically important mineral deposits, including clays, carbonates, serpentine and sulfates, with analysis of the petrology to understand their formation environment, such as surface weathering, lake sediments or hydrothermal activity. Tighter quantification of volatile reservoirs, isotopic measurements to constrain early geochemical cycles, and radiometric dating of a range of deposits to provide absolute age constraints are also vital for future progress ${ }^{7}$.

Fortunately, we are on the verge of a resurgence in the in situ exploration of Mars. In 2020, the European Space Agency's ExoMars mission and China's 2020 mission will both carry surface rovers in their payloads. NASA's Mars 2020 rover, which is currently undergoing final landing site selection, will both conduct in situ science on the surface and cache samples for a future sample return mission. The international effort to return samples of known geologic context to Earth for comprehensive chronological, geochemical and mineralogical analysis holds the promise of initiating a revolution in our understanding of Mars' early history.

The early evolution of Mars is one of the most fascinating and inspiring problems in planetary science. Far from being 'at war', our field is currently developing rapidly, with significant advances over the last decade occurring in both observations and modelling. By studying Mars, we all gain insight not just into one of our home planet's closest neighbours, but also into planetary habitability in general. As we develop the tools to search for life on exoplanets and continue to investigate other objects in the solar system, the Red Planet, which is so strangely Earth-like in some ways and yet so utterly alien in others, must remain a key research and exploration target.

\section{R. Wordsworth ${ }^{1,2 \star}$, B. EhImann ${ }^{3,4}$, F. Forget ${ }^{5}$, R. Haberle ${ }^{6}$, J. Head' ${ }^{7}$ and L. Kerber ${ }^{4}$ ${ }^{1}$ School of Engineering and Applied Sciences, Harvard University, Cambridge, MA, USA. ${ }^{2}$ Department of Earth and Planetary Sciences, Harvard University, Cambridge, MA, USA. ${ }^{3}$ Division of Geological and Planetary Sciences, California Institute of Technology, Pasadena, CA, USA. ${ }^{4}$ Jet Propulsion Laboratory, California Institute of Technology, Pasadena, CA, USA. ${ }^{5}$ Laboratoire de Météorologie Dynamique, Institut Pierre Simon Laplace, Paris, France. ${ }^{6}$ Planetary Systems Branch, NASA Ames, Mountain View, CA, USA. ${ }^{7}$ Department of Earth, Environmental and Planetary Sciences, Brown University, Providence, RI, USA. *e-mail: rwordsworth@seas.harvard.edu}

Published online: 30 November 2018 https://doi.org/10.1038/s41561-018-0267-5

\footnotetext{
References

1. Nat. Geosci. 11, 219 (2018).

2. Wordsworth, R. D. Annu. Rev. Earth Planet. Sci. 44 381-408 (2016)

3. Haberle, R. M., Clancy, R. T., Forget, F., Smith, M. D. \& Zurek, R. W. The Atmosphere and Climate of Mars (Cambridge Univ. Press, Cambridge, 2017).

4. Hoke, M. R. T., Hynek, B. M. \& Tucker, G. E. Earth Planet. Sci. Lett. 312, 1-12 (2011).

5. Grotzinger, J. P. et al. Science 343, 1242777 (2014)

6. Fastook, J. L. \& Head, J. W. Planet. Space Sci. 106, 82-98 (2015).

7. Ehlmann, B. L. et al. J. Geophys. Res. Planets 121, 1927-1961 (2016).
} 